\title{
UNA NUEVA ESPECIE DE GRIPOPTERYGIDAE DE CHILE, POTAMOPERLA TESTACEA N. SP. (INSECTA: PLECOPTERA)
}

\section{NEW SPECIES OF GRIPOPTERYGIDAE OF CHILE, POTAMOPERLA TESTACEA N. SP. (INSECTA: PLECOPTERA)}

\author{
Alejandro Vera \\ Universidad Metropolitana de Ciencias de la Educación, Casilla 417, Santiago. \\ Email: alveras2@gmail.com
}

\begin{abstract}
RESUMEN
Se describe una nueva especie de Gripopterygidae: Gripopteryginae (sensu McLellan 1977) para Chile, Potamoperla testacea n. sp. distribuida entre las regiones Séptima y Undécima. Se dan a conocer ambos sexos y la ninfa, esta última asociada mediante emergencias de laboratorio. Se discute su ubicación genérica.

Palabras claves: Taxonomía, Plecoptera, nueva especie, Gripopterygidae, Potamoperla, ninfa.
\end{abstract}

\section{ABSTRACT}

A new species of Gripopterygidae: Gripopteryginae (sensu McLellan described 1977) from Chile Potamoperla testacea n. sp. distributed between the Seventh and Tenth first Regions. Is both sexes and the nymph are given to know, this last one is associated by means of laboratory emergence. Its generic location is discussed.

Keywords: Taxonomy, Plecoptera, new species, Gripopterygidae, Potamoperla, nymph.

\section{INTRODUCCION}

Illies (1963) crea el género Potamoperla en base a Perla myrmidon Mabille, 1891 y sinonimiza las especies Gripopteryx oncina y Gripopteryx michelbacheri, ambas descritas por Fröehlich (1960). De esta manera el género queda establecido como monoespecífico y distribuido desde los $32^{\circ}$ (Mendoza, Argentina) hasta los $53^{\circ}$ (Tierra del fuego, Magallanes, Chile) de latitud sur. Illies (op. cit.) describe la ninfa en base a especímenes colectados en Tierra del Fuego, algunos de ellos correspondían a ninfas de último instar macho, que mediante extracción de las piezas genitales del adulto contenidas en su interior, fueron asociadas a este género.

El abundante material revisado por Illies (80 hembras y 105 machos) le permitió establecer el patrón constante de pequeñas manchas irregulares que cubren todo el primer par de alas en ambos sexos y los rangos de variación en la genitalia masculina. En el presente estudio se describe una nueva especie de este género, $P$. testacea, que difiere en los rasgos antes mencionados por Illies, pese a que su distribución está incluida en el rango de la especie preexistente, además se describe la ninfa cuyo hábitat y morfología la distinguen claramente de $P$. myrmidon.

\section{MATERIALES Y METODOS}

El material estudiado de Potamoperla testacea se encuentra depositado en la Colección del Museo Nacional de Historia Natural (Santiago, Chile), conservado en alcohol de $70 \%$ (salvo cuando se indica: en preparación microscópica), las 
localidades y el número de ejemplares se indican a continuación de la descripción, junto con designar el material tipo. El material de Potamoperla myrmidon utilizado para las comparaciones se encuentra depositado y conservado en las mismas condiciones que la especie anterior, y se detalla como sigue: Chile VI Región, Río los Cipreses 6-IV-1996, col. A. Vera (3 ninfas, $20^{\star}$ ); VII Región, Vilches Alto

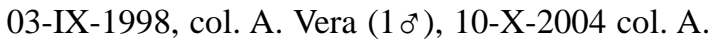
Vera (2 +); Río Curanilahue sector Los Ruiles 30IV-2005, col. A. Vera (1 ninfa); IX Región, Curacautín Manzanar, 22-IX-2004 col. A. Vera (1 ㅇ); X Región, Chiloé, 16-II-2002, leg. M. Guerrero (2우, $\left.20^{\top}\right)$.

La descripción de los adultos y la ninfa se realizó siguiendo la nomenclatura utilizada por McLellan (1971). Las disecciones y mediciones corporales se realizaron bajo lupa estereoscópica Nikon con oculares de 10X y objetivos (zoom) de $0,7 \mathrm{X}$ a $3 \mathrm{X}$, Los dibujos fueron realizados bajo cámara clara en microscopio Leitz. Dialux 22Bauchamp-loop. Los ejemplares montados en preparaciones microscópicas fueron tratados con $\mathrm{KOH} 10 \%$ sin calentar por $12 \mathrm{~h}$. y montados en solución Doetschman coloreados con lignina rosada (Camousseight \& Fontaine 1990).

Sólo se cuenta con un adulto emergido en laboratorio desde una ninfa de último instar. Las condiciones de crianza fueron: temperatura entre 5 a $7^{\circ} \mathrm{C}$, con bomba de aire, renovación de agua cada dos semanas durante 2 meses, alimentación con hojas de Notofagus alpina (tanto el agua como las hojas fueron tomadas y refrigeradas desde el sitio de muestreo). El adulto fue mantenido vivo por $24 \mathrm{~h}$., para lograr su total esclerosis, antes de ser fijado en alcohol de $70 \%$.

\section{RESULTADOS}

Descripción adultos Potamoperla testacea n. sp. (Fig. 1- 4)

Longitudes medias $\pm s$ en mm.:

$\sigma^{7} \mathrm{n}=5$ : cuerpo 7.14 \pm 0.31 ; ala anterior $9.52 \pm 0.0$; cuerpo con alas $11.26 \pm 0.31$; antenas $6.19 \pm 0.18$; cercos $1.18 \pm 0.16$.

i $\mathrm{n}=7$ cuerpo $8.67 \pm 0.96$; ala anterior $10.9 \pm 0.71$; cuerpo con alas $12.31 \pm 0.72$; antenas $6.29 \pm 0.42$; cercos $1.26 \pm 0.0$.
Coloración general testáceo a amarillento.

Cabeza (Fig. 1): frente trapezoidal, tres ocelos equidistantes, ojos prominentes, occipucio ligeramente más oscuro que el resto de la cabeza, borde dorsal en la articulación de las antenas negro, suturas amarillentas, región media dorsal levemente deprimida. Antenas de 30 artículos, con abundante micropilosidad negra.

Protórax (Fig. 1): transversal 0,25 veces más ancho que largo, de lados rectos y paralelos, ángulos redondeados, margen anterior recto, ligeramente inclinado hacia posterior en los extremos, margen posterior emarginado medialmente. Región central convexa y rugosa, línea media hundida, bordes laterales y anterior aplanados y lisos.

Patas: Tibias con cuarto proximal ennegrecido, tarsos marrón y garras negras, el resto testáceo. Primer par de patas con fémures claros, tibias oscuras en ambos extremos, segundo y tercer par de patas con fémures más oscuros que las tibias. Relación de la longitud de los tarsos 4:3:10.

Alas (Fig. 2): de ápice ligeramente puntiagudo, lámina hialina a testácea. Ala anterior, con características manchas oscuras cuadrangulares en cada vena transversal, área entre C y R Marrón rojizo, en algunos casos hasta Rs. Número de venas transversales entre $\mathrm{R} \mathrm{y} \mathrm{Cu} 2$ (no incluye las tres venas basales entre $\mathrm{M}$ y Cu1): Machos: 3-4:3-4:3-5:3-4:12:9-10, Hembras: 3-4:(0-1):4-5:3-4:3-4:1-2:8-11. Sólo una hembra presenta venas transversales en inusual número: 1 en la orquilla de Rs y 11 entre Cu1 y Cu2. La bifurcación de Rs es corta, la bifurcación de Cu1 es larga. Ala posterior, con pterostigma hialino a marrón rojizo, con bifurcación corta de Rs y Cu1.

AвDomen: Testáceo, manchado irregularmente con tonos más oscuros. Tergos I al VIII con mitad anterior esclerosada, esta placa alcanza lateralmente el borde posterior y presenta una delgada línea media dorsal no esclerosada entre I a IV ocasionalmente hasta VIII. Esternos del I al VII sólo con un par de placas esclerosadas en el margen anterior. En los machos esterno VIII con placa medio posterior trapezoidal y un par de placas en los ángulos anteriores. En hembras segmentos del I al VI sin regiones esclerosadas.

\section{Genitalia masculina (Fig. 3):}

TERgo X: Escleritos anteriores, laterales y central fundidos. Margen posterior apicalmente proyectado en una única punta aguda muy esclerosada, 
ensanchada en su base y orientada ventralmente en ángulo de $90^{\circ}$ respecto de la placa tergal (probablemente derivado del esclerito posterior). En la línea media dorsal se dispone una región elipsoide poco o nada esclerosada que varía pudiendo igualar la longitud del tergo. Un par de pequeños escleritos redondeados próximos a la línea media se proyectan hacia el borde anterior del tergo. Con una ligera prominencia aguda (evidente en vista dorsal), en el borde de la articulación de los cercos. Epiprocto: ausente. Paraproctos: largos superando la longitud del tergo $\mathrm{X}$, curvados dorsalmente $\mathrm{y}$ encontrándose distalmente, con una lámina plana de ápice truncado en su extremo. Placa subgenital ligeramente más larga que ancha, estrechándose hacia la base, borde posterior recto con ángulos redondeados. Cercos: de 11 segmentos con pilosidad corta.

Genitalia femenina (Fig. 4): Placa subgenital corta, no logra cubrir el esterno siguiente, borde posterior recto, ligeramente emarginado, fuertemente esclerosado, con un par de regiones triangulares proyectadas hacia el borde anterior, que pueden llegar a fundirse formando una gran placa rectangular, pilosidad corta y curva, de densidad variable. Tergo $\mathrm{X}$ puntiagudo, con lóbulos subanales delgados, con su mitad basal más ancha, de ápice puntiagudo, se prolongan y finalizan junto al tergo $\mathrm{X}$.

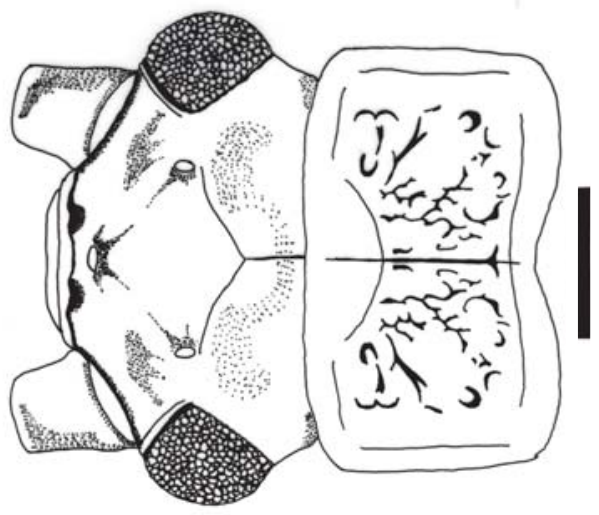

Figura 1. Cabeza y tórax de macho (escala 0.3mm).

FIGURE 1. Male head and thorax (scale 0,3mm)

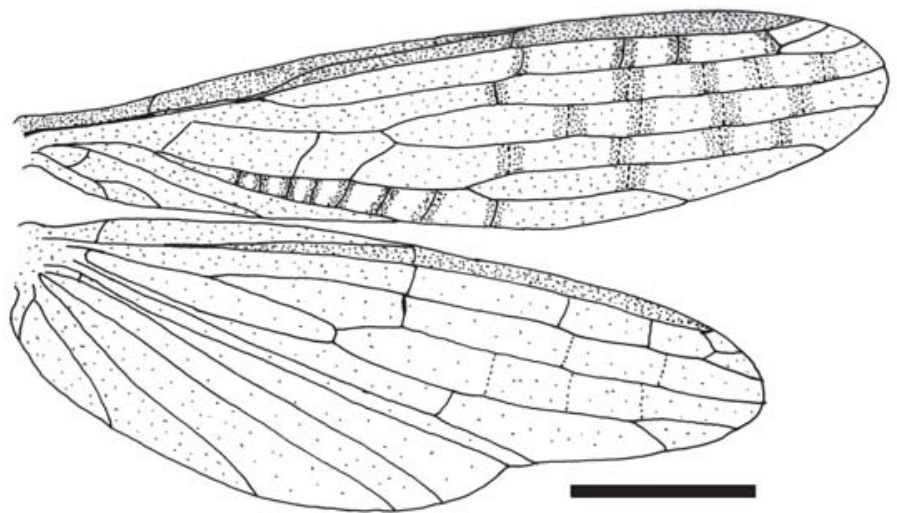

Figura 2. Alas macho (escala 2mm).

FIGURE 2. Male wings (scale 2mm). 
Un nuevo Gripopterygidae de Chile. Vera, A.
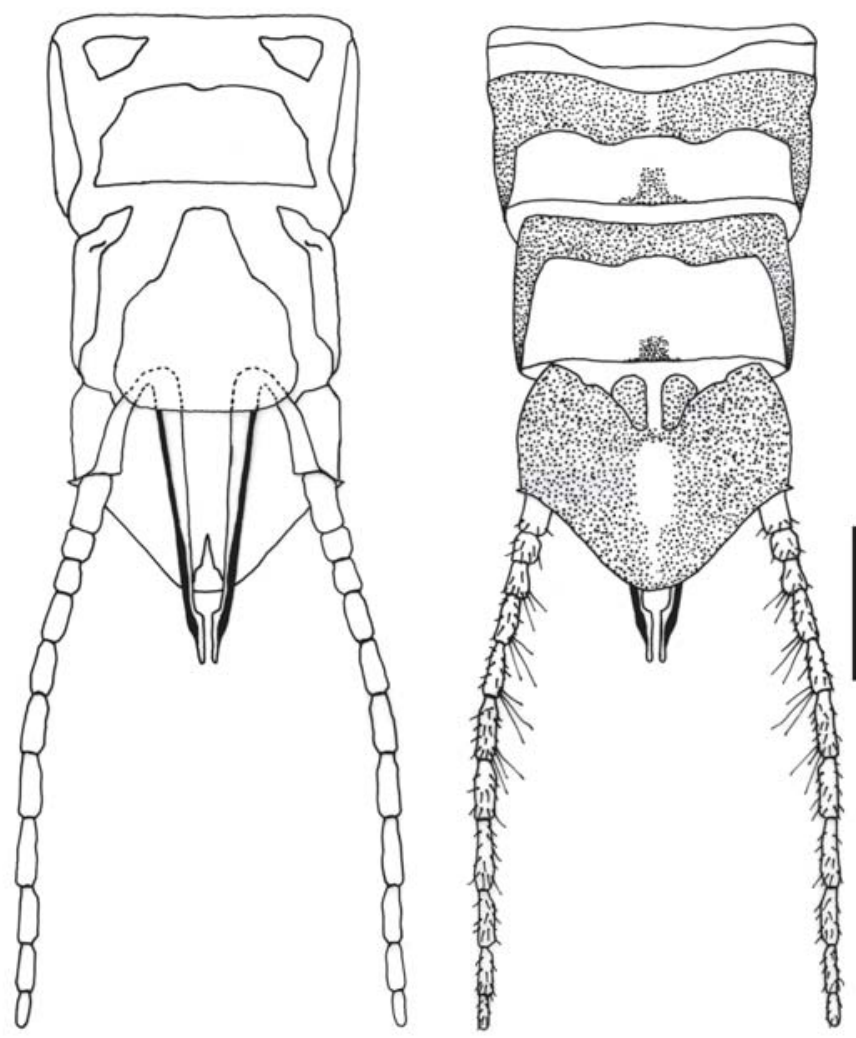

Izquierda/ Left

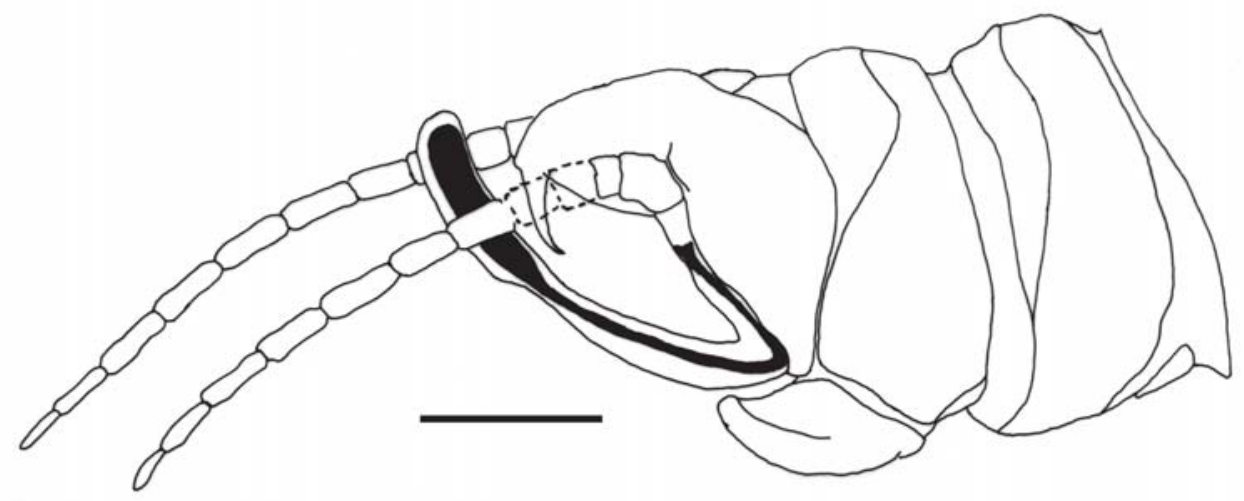

Derecha/ Right

FIGURA 3. Genitalia masculina y últimos segmentos abdominales de izquierda a derecha: Vista ventral, dorsal y lateral (escala $0.3 \mathrm{~mm}$ ).

FIgURE 3. Male genitalia and last abdominal segments: Top ventral and dorsal view, below lateral view (scale 0,3mm). 


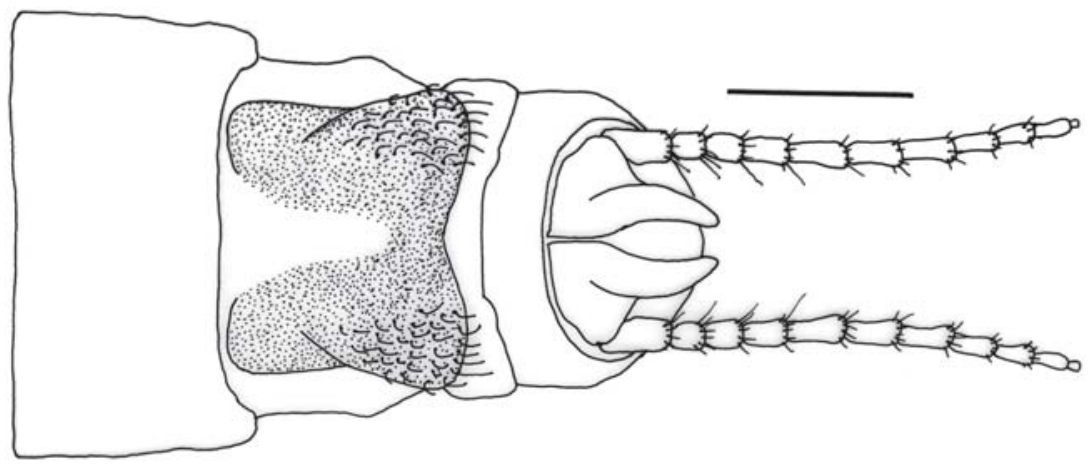

Figura 4. Ultimos segmentos abdominales de la hembra en vista ventral (escala 0.5).

Figure 4. Last abdominal segments of the female in ventral view (scale 0,5).

DESCRIPCIÓN NINFA ÚLTIMO INSTAR (Figs. 5-10):

Longitudes medias $\pm s$ en mm.: $\mathrm{n}=2$ cuerpo $6.98 \pm$ 0.03 ; antenas $3.69 \pm 0.4$; cercos $0.94 \pm 0.12$.

Cuerpo de aspecto rígido, cilíndrico, color general marrón oscuro con antenas, cercos y tarsos amarillentos. Tegumento areolado con micropilosidad densa y en su mayor parte homogéneamente distribuida.

CABEzA: sin manchas con tres ocelos, antenas glabras, sólo con un anillo de pequeñas cerdas subapicales, cuya longitud es la mitad del artejo respectivo, únicamente visibles bajo microscopio. Armadura bucal (Figs. 6-8 y 10): mandíbulas (Fig. 8), ambas con un largo mechón de pelos, cuya longitud es semejante a la región molar y se ubican por debajo de ésta. Maxilas (Fig. 6), palpo maxilar de 4 artejos, el último notablemente más largo que los anteriores, cilíndrico y ligeramente puntiagudo, longitud de los segmentos según la siguiente proporción: 5:5:10:22. Labro (Fig. 10) rectangular, apicalmente convergente y con un pequeño lóbulo medio en el borde anterior. Labio (Fig. 7) tercer segmento del palpo labial de ápice redondeado, tanto o más largo que la suma de los dos anteriores. Tórax (Fig. 5): con regiones ennegrecidas según el diseño que muestra la figura. Pronoto cuadrado, de lados rectos ligeramente divergentes anteriormente, borde anterior recto y ligeramente convexo, borde posterior recto, ángulos redondeados, región central convexa ligeramente rugosa con línea media hundida, márgenes anterior y laterales lisos.
Mesonoto cuadrado, plano, borde posterior recto, márgenes laterales expandidos y cubriendo parcialmente los estuches de las alas en su porción basal. Metanoto plano, con borde posterior proyectado ligeramente en dos ápices redondeados. Estuches de las alas anteriores dispuestos lateralmente, estuches de las alas posteriores dispuestos dorsolateralmente, ambos estuches con ligeras líneas longitudinales de cerdas, todo su contorno con una fila continua de pequeñas cerdas puntiagudas.

Patas cortas y robustas, glabras, con tenues bandas más oscuras en el extremo distal de los fémures y la base de las tibias. Tarsos: longitud de sus segmentos según la siguiente proporción 2:3:10; tercer par de patas 1:1:6.

Aвdomen (Figs. 5 y 9): tergos (Fig. 5) del I al IX con un par de pequeñas manchas dorsales, hacia el extremo estas manchas se van alargando, a partir del tergo VI forman bandas longitudinales, y aparece una notoria banda media, tergo $\mathrm{X}$ mayoritariamente negro, de borde posterior puntiagudo; lóbulos subanales (Fig. 9) con forma de lengüeta, anchos, de ápice truncado, sobrepasan levemente el tergo $\mathrm{X}$. Cercos cortos (tan largos como los últimos dos tergos) y glabros con finas cerdas subapicales a cada artejo que no superan la longitud del siguiente, éstas son visibles sólo bajo microscopio. Ramillete de branquias anales de longitud semejante al tergo $\mathrm{X}$. borde posterior de los tergos I al IX con fila continua de cerdas puntiagudas, visibles bajo microscopio. 


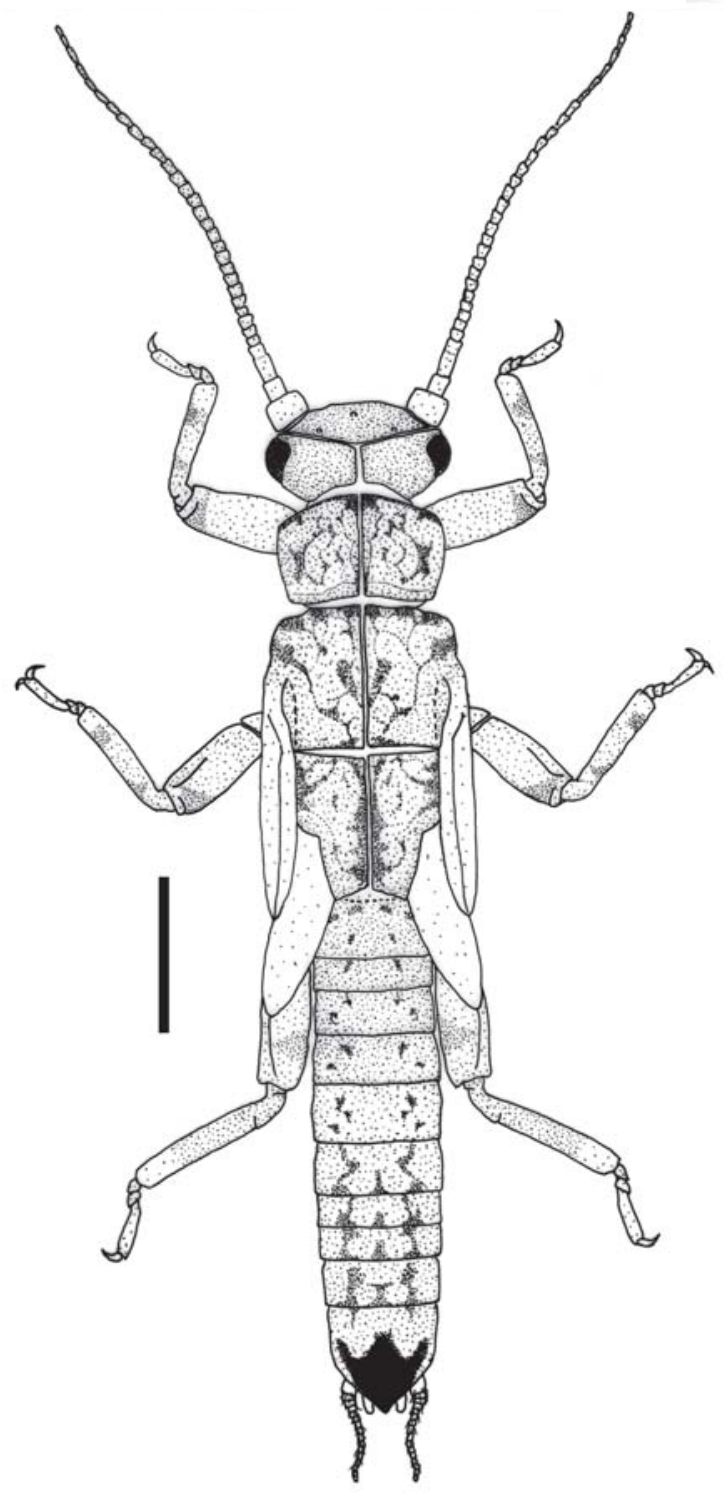

Figura 5. Ninfa vista dorsal (escala $0.1 \mathrm{~mm}$ ), se omiten las branquias.

FIGURE 5. Nymph dorsal view (scale 0,1mm), the gills are omitted.

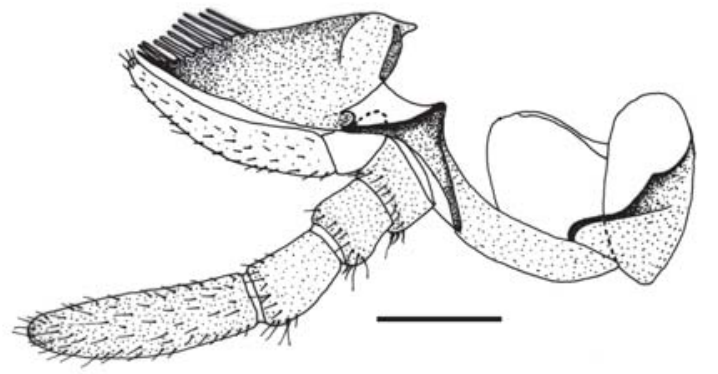

Figura 6. Maxila (escala 0.1mm).

Figure 6. Maxilla (scale 0,1mm).

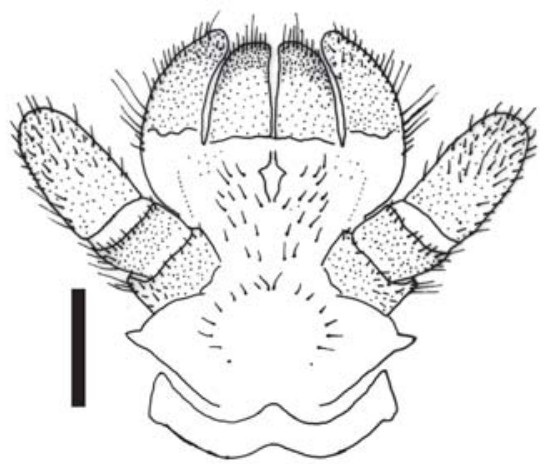

Figura 7. Labio (escala $0.1 \mathrm{~mm}$ ).

Figure 7. Labium (scale 0,1mm).

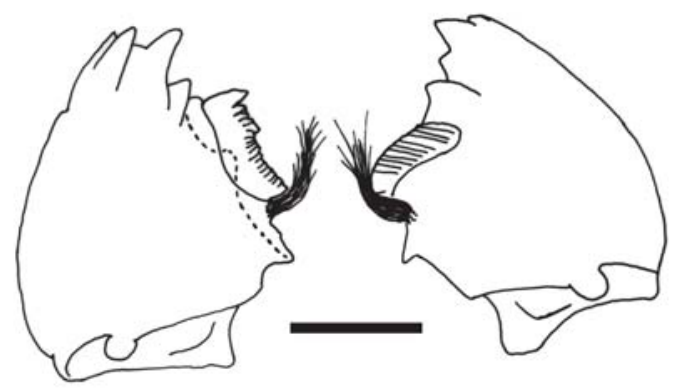

Figura 8. Mandíbulas (escala 0.1mm).

FIGURE 8. Mandibles (scale 0,1mm). 


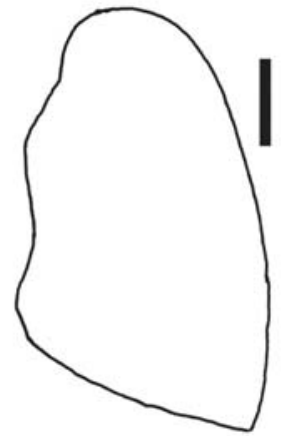

Figura 9. Lóbulo subanal (escala 0.1mm).

Figure 9. Subanal lobe (scale 0,1mm).

MATERIAL TIPO

Holotipo: $\sigma^{*}$, Chile IX Región, Curacautín Manzanar, 22-IX-2004, col. A. Vera.

Alotipo: ㅇ, Chile IX Región, Malleco, Termas de Tolhuaca, 5-II-2001, leg M. Guerrero.

PARATIPOs: 7 o y 5 ơ (1 de ellos con su exuvia ninfal) con los siguientes datos: Imagos: leg. M. Guerrero; Chile IX Región, Malleco, Río Blanco, 9-II-2000 (1ㅇ, 10`); Termas de Tolhuaca, 12-II-1998 (3우). Col. A. Vera; Chile VII Región, Río Curanilahue, sector Los Ruiles (arrollo), 26-VIII-2004 (2 $\sigma^{*}$, uno de ellos en preparación microscópica); IX Región, Lonquimay, estero El Durazno, emerge 30-XII-2004 $\left(10^{\star}\right)$, Parque Nacional Conguillío, estero Carpintero 20-I-2005 (2 + y 1 $0^{\star}$ ); XI Región Coyhaique, Valle Soler 20-I-1998 (1 ㅇ).

Ninfas: exuvias de emergencia (en preparación microscópica): Chile VII Región, Río Curanilahue, sector Los Ruiles 13-IX-2005 (2, tomadas desde terreno, una incompleta); IX Región, Lonquimay estero El Durazno, emerge en laboratorio XII-2004 $\left(10^{\top}\right)$.

\section{ETIMOLOGÍA}

El nombre de la especie es un adjetivo femenino en concordancia con el género, se debe a su particular coloración testácea, que permite identificarlo a simple vista en condiciones de terreno, destacándose entre otras especies acompañantes.

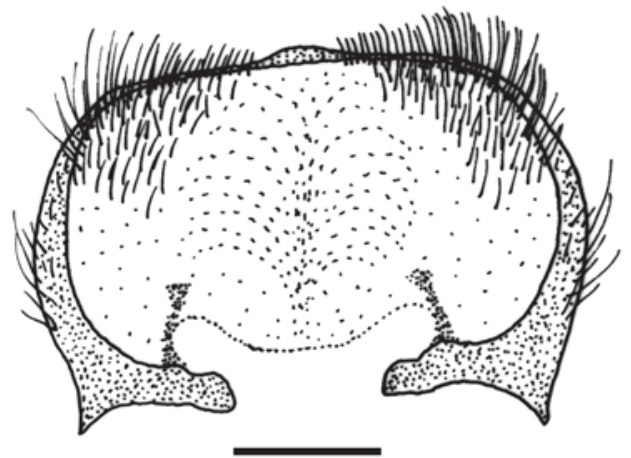

FiguRA 10. Labro (escala $0.1 \mathrm{~mm}$ ).

Figure 10. Labrum (scale 0,1mm).

HÁBITAT Y DISTRIBUCIÓN

Las ninfas se encuentran en esteros muy fríos, de corriente rápida y entre rocas sin acumulación de sedimentos ni musgos. Los adultos han sido colectados sobre el follaje asociado a esteros de semejantes características, tanto el la Cordillera de los Andes como en la Cordillera de la Costa, entre las regiones VII y XI (354'ㅇ's a 46 $59^{\circ} S$ ).

\section{DISCUSION}

Se incluye esta especie en el género Potamoperla, fundamentalmente por presentar los siguientes caracteres idénticos al género en los adultos: pronoto transversal de ángulos redondeados, idéntica nervadura alar salvo por la bifurcación de Rs corta en el primer par de alas, placa subgenital para ambos sexos, longitud de los cercos, tergo $\mathrm{X}$ de la hembra puntiagudo, tergo $\mathrm{X}$ del macho con los escleritos fundidos, paraproctos alargados y de semejante disposición, epiprocto ausente siendo este último carácter compartido únicamente con el género Paragripopteryx Enderlein, propio de la costa atlántica de Brasil.

Para identificar los imagos de la nueva especie respecto de $P$. myrmidon son considerados caracteres diagnósticos en ambos sexos la coloración general testácea y el patrón regular de manchas en las venas transversales de las alas anteriores; en la genitalia 
masculina el ápice del tergo $\mathrm{X}$ proyectado en una única espina puntiaguda dispuesta en $90^{\circ}$ respecto del tergo.

La ninfa de $P$. testacea se distingue de $P$. myrmidon en que esta última presenta 5 segmentos en los palpos maxilares; su cuerpo es blando y estilizado; con patas, cercos y antenas alargadas; todo el cuerpo cubierto de largos y finos pelos, especialmente cercos, antenas y patas (Illies indica que la pilosidad le permite adherir restos de barro como camuflaje), los cercos al menos son tan largos como los últimos 8 tergos, la ninfa de $P$. testacea, tal como se indica en la descripción, es mucho más robusta, de cuerpo más esclerosado, de particular diseño abdominal, de patas, cercos y antenas cortas, las cerdas que cubren su cuerpo son diminutas y en sus apéndices son escasas. Respecto del hábitat, las ninfas $P$. myrmidon se desarrollan en corriente lenta con sedimento fangoso, mientras que $P$. testacea se ha encontrado en corriente lótica con escaso sedimento. La diagnosis genérica en cuanto a las ninfas es compleja, sólo gracias a la asociación lograda mediante emergencia de laboratorio, es posible incluir en Potamoperla la ninfa de la nueva especie, su descubrimiento independiente del adulto pondría en duda su ubicación genérica. Dentro de la familia Gripopterygidae no existen casos de tal discrepancia en la morfología ninfal de especies congenéricas, este hallazgo es aún más relevante si se considera que en el $50 \%$ de las especies citadas para Chile las ninfas son desconocidas.

En cuanto a la distribución de la nueva especie, se encuentra incluida en la distribución de la especie preexistente.

\section{AGRADECIMIENTOS}

Agradezco al Dr. Ariel Camousseight por facilitar parte de la literatura utilizada, por su lectura crítica del manuscrito y su constante estímulo en el estudio de estos insectos. Al Museo Nacional de Historia Natural, Entomología, por facilitar laboratorios y equipos que hicieron posible este estudio.

\section{BIBLIOGRAFIA}

Camousseight, A. \& J. Fontaine. 1990. The biological cycle of Baetis pentaphlebodes, Ujhelyi 1966, in an old meander of the Rhone river, france (Ephemeroptera: Baetidae). I. C. Campbel (ed.) Mayflies and Stoneflies, 27-34. Kluwer Academic Publishers, Dordrecht.

Fröenlich, C.G. 1960. Some Gripopterygids and Notonemourines (Plecoptera) from South America. Lunds universitets Arsskrift (N.F. Abt. 2) 56(13):1-23.

Illies, J. 1963. Revision des südamerikanischen Grypopterygidae (Plecoptera). Mitteilungen der Schweitzerischen Entomologischen Gesellschaft 36 (3): 145-248.

Mabille, P. 1891. Néuroptères. En: Zoologie Missions Scientifique du Cap Horn 1882-1883, 6(2) D III:6-9.

McLellan, I.D. 1971. A revision of Australian Gripopterygidae (Insecta: Plecoptera). Australian Journal of Zoology: Supplementary Series $\mathrm{N}^{\mathrm{o}}$ 2.pp:1-79.

McLellan, I. D., 1977: New alpine and southern Plecoptera from New Zealand and a new classification of the Gripopterygidae. New Zealand Journal of Zoology 4:119-147. 